\title{
Effects of Cognitive Remediation Therapy on working memory and school results in children with autism spectrum disorder
}

\author{
Hajri M., Abbes Z., Ben Yahia H., Taamallah.A, Halayem S., Bouden A. \\ Child and Adolescent Psychiatry Department- Razi University Hospital - Manouba - \\ Tunisia
}

\section{Introduction:}

Autism spectrum disorder (ASD) is known to be associated with cognitive dysfunctioning, involving in particular working memory

[1]. This impairment has an impact on academic difficulties and it is one of the treatment targets. Cognitive remediation therapy (CRT) attempts to improve cognitive deficits by teaching information processing strategies through guided mental exercises [2].

\section{Objectives:}

The aim of this study is to investigate whether CRT, a new psychological treatment, improves working memory ad school performance in children with autism spectrum disorder.

\section{Methods:}

Children meeting the Diagnostic and Statistical Manual for Mental Disorders (DSM-5) criteria for ASD, and following a regular school curriculum were recruited from clinical population at the Child and Adolescent Psychiatry Department in Razi University Hospital - Manouba - Tunisia. The intervention was individual CRT, delivered over a period of six months with one 45-minute session per week. Working memory was assessed using forward and backward digit span. School performance was assessed by school results.

\section{Results:}

Of the 25 patients included, 16 achieved assessments tests after CRT, thus constituting our final sample. Their average age was 10.87 years. The mean number of sessions performed was 22.38

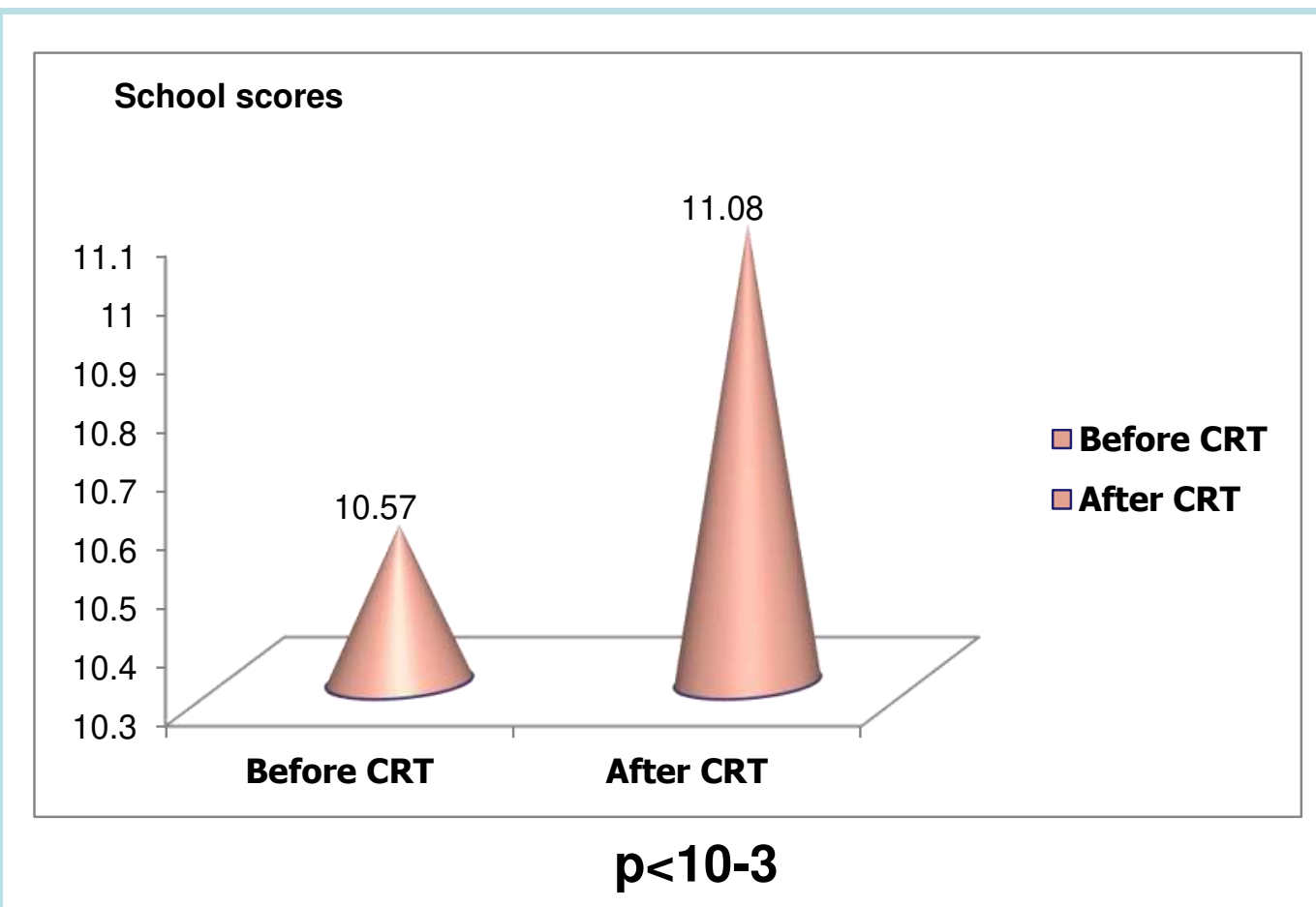

Chart 1: Comparison of school results before and after CRT

\section{Discussion:}

$>$ A small number of researches studied the use of CR in ASD. In our study, patients significantly improved in forward and backward digit span. Our findings are in line with literature data [3,4,5]. In fact, Tan et al conducted a study in 2013 [3], investigating the efficacy of $\mathrm{CR}$ in alleviating cognitive deficits in patients with schizophrenia. Results showed a significant effect of CR in working memory assessed by the Wechsler Adult Intelligence Scale (WAIS)-Digit Span Forward and Backward. This result is particularly interesting because working memory is highly related to social functioning in patients with schizophrenia [6].

$>$ Regarding school results, a significant improvement was noticed. Indeed, the benefits of CR translate to practical gains for the participants. In fact, school tasks include reasoning and problem solving exercises that have already been trained throughout the CRT program, mainly in planning session. This improvement in school results could also be due to improved self-esteem. In line with our findings, Tan et al found that patients with schizophrenia displayed significantly greater improvement and better vocational skills after a CR program [7]. Conclusion:

The cognitive remediation Therapy is a promising technique that seems to be effective in the management of children with autism spectrum disorder.

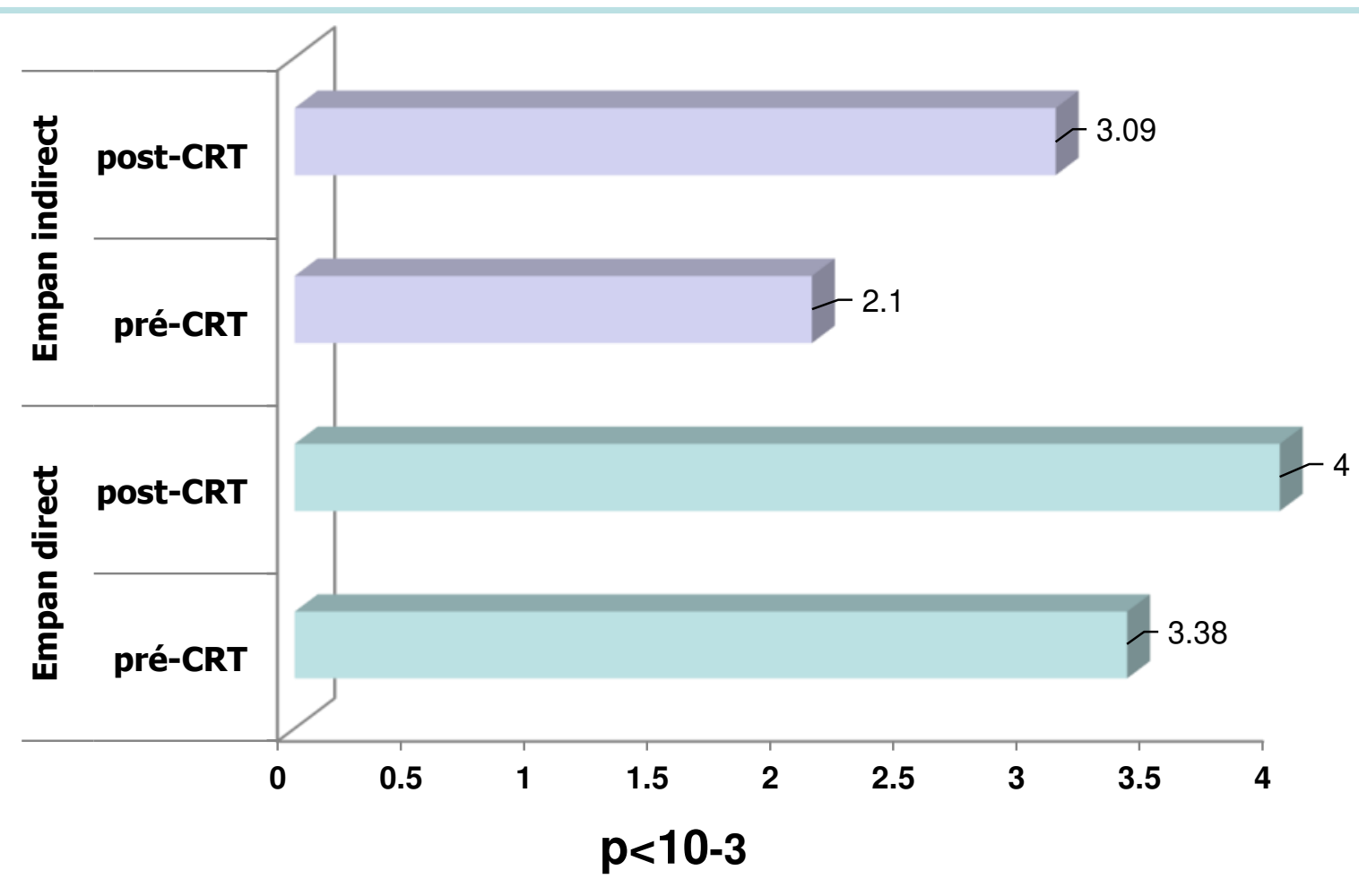

Forward digit span: $p=0,001$

Backward digit span: $p=0,006$

Chart 2: Comparison of forward and backward digit span before and after CRT

\section{References:}

